\title{
Multiple-Gap Theory of Toroidal Alfvén Waves with Kinetic Effects
}

\author{
X.D. Zhang, Y.Z. Zhang, and S.M. Mahajan \\ Institute for Fusion Studies \\ The University of Texas at Austin \\ DOE/ET/53088--573 \\ DE93 001852 \\ Austin, Texas 78712
}

\begin{abstract}
The stability of kinetic toroidal Alfven waves with multi-gap coupling is analyzed by using the two-dimensional ballooning transform. An alternate convergence scheme, based on the smallness of the inverse aspect ratio, is devised. The resulting wave functions are oscillatory and do not balloon in contrast to the wave functions of conventional ballooning theory. It is shown that the single-gap theory is a special, weak shear $(s \rightarrow 0)$ limit of the formalism. Analytical and numerical results for the two fundamental branches, the ideal toroidal Alfvén eigenmode (TAE), and the kinetic toroidal Alfvén eigenmode (KTAE) are presented and discussed.
\end{abstract}




\section{Introduction}

The toroidal Alfvén eigenmodes (TAE) received great attention recently, because of their possible importance for burning plasma experiments. ${ }^{1-11}$ It has been suggested that these modes are likely to be excited by fusion alpha particles, ${ }^{5}$ and could cause deterioration in alpha particle confinement, and hence of the self-sustained heating efficiency. In the light of recent experiments, ${ }^{5}$ which showed that the TAE instability thresholds may be much larger than the prediction of the earlier theories, it is crucial that a thorough investigation should be made of all the possible damping mechanisms present in the plasma. Since then, within the framework of the ideal magnetohydrodynamics, much effort has been spent on the calculation

of damping rates due to Alfvén resonance; the so-called continuum damping. ${ }^{6-8,10}$ Very recently, however, investigations of kinetic effects on TAE revealed the intrinsic importance of the combination of toroidicity and kinetics for Alfvén waves. ${ }^{11}$

A novel branch, the so-called kinetic toroidal Alfvén eigenmode (KTAE), emerges from the continuum due to electron kinetics; this mode smoothly goes over to the well-known kinetic Alfvén eigenmode in the slab limit. ${ }^{12-14}$ The electron kinetics also cause a strong stabilization of the ideal TAE. The kinetic effects on the stability of both the KTAE and the TAE (with a well-defined ideal limit) have been carried out in Ref. 11, where the theoretical framework is built around a single gap. This assumption seems to be plausible for very low toroidal mode number $(n)$ and small magnetic shear, for which the adjacent gaps are well separated, and the tunneling would be insufficient to induce a strong coupling between the gaps. However, for either moderate $n$-number and/or moderate magnetic shear, the coupling of gaps could be quantitatively significant. It is the purpose of this paper to evaluate the multi-gap effects on these two fundamental branches, the KTAE and the TAE.

A multiple-gap calculation is fundamentally a two-dimensional calculation, and must in- 
voke an intrinsic toroidal coupling scheme. The conventional ballooning theory ${ }^{15-18}$ (with its quota of successes in dealing with the ideal system) encounters the problem of a complex solvability condition for non-ideal systems. ${ }^{19,20}$ Taking advantage of an additional small parameter, the inverse of the large aspect ratio, an alternate scheme is devised for the intrinsic toroidal coupling. The scheme is based on two-dinıensional (2D) ballooning transform, ${ }^{19}$ and exhibits several distinctive features. Among others, the eigenvalue of the $2 \mathrm{D}$ system is determined as an average over the eigenvalues of the parameterized ballooning equation $z \mathrm{~s}$ a consequence of the periodicity constraint imposed on the 'non-ballooned' wave function. In the conventional ballooning theory, on the other hand, the $2 \mathrm{D}$-eigenvalue is determined by a local parameterized ballooning eigenvalue at the extremum, e.g. $\lambda=0$, or $\lambda=\pi .^{15-20}$ The necessary details of the formalism are given in Sec. II, while in Sec. III the analytic and numerical results are presented for the two fundamental branches. Comparison with the single-gap results will be discussed in Sec. IV, where we also show how the basic equation for the single-gap theory of Ref. 11 can be obtained from the ballooning formalism.

\section{Ballooning Theory for Toroidal Alfvén Waves}

The 2D eigenmode equation describing the Alfvén waves in an axisymmetric tokamak with circular cross-section is modelled by

$$
\begin{gathered}
\frac{d}{d x}\left[\frac{\Omega^{2} f(x)}{4}-(x-\ell)^{2}\right] \frac{d \phi_{\ell}}{d x}-\frac{1}{s^{2}}\left[\frac{\Omega^{2} f(x)}{4}-(x-\ell)^{2}\right] \phi_{\ell} \\
+\epsilon \frac{\Omega^{2}}{4}\left(\frac{d^{2} \phi_{\ell+1}}{d x^{2}}+\frac{d^{2} \phi_{\ell-1}}{d x^{2}}\right)+\hat{b}\left(\frac{d^{2}}{d x^{2}}-\frac{1}{s^{2}}\right)^{2} \phi_{\ell}=0
\end{gathered}
$$

where $\phi_{\ell}$ is the Fourier component defined by the physical electrostatic potential $\Phi(r, \theta, \zeta) \equiv$ $\exp (i n \zeta-i m \theta) \sum_{\ell} \exp (-i l \theta) \phi_{\ell}(x), x \equiv n\left(q-q_{0}\right)$ labels the radial position, $\theta(\zeta)$ is the poloidal (toroidal) angle, $m$ is the leading poloidal mode number, $q_{0} \equiv m / n, s \equiv(d \ell n q / d \ell n r)_{q=q_{0}}$ is the magnetic shear, $\Omega \equiv \omega / \omega_{A}$ is the mode frequency normalized to the Alfvén frequency 
$\omega_{A} \equiv v_{A} /(2 R q), v_{A}$ is the Alfvén speed, $f(x)$ represents the radial variation of the Alfven speed, $\epsilon \equiv 5 r / 2 R$, is the toroidal coupling constant, $R(r)$ is the major (minor) radius, $\hat{b}$ stands for the non-magnetohydrodynamic effect (primarily the electron parallel response) measured by $\left(\rho_{s} k_{\theta} s\right)^{2} / 4$ plus an imaginary part representing the electron Landau damping, and $k_{\theta} \equiv m / r$ is the poloidal wave number.

The physical meaning of individual terms in Eq. (1) is transparent: the first two constitute magnetic bending and ion inertia; and the remaining represent the toroidal coupling, and the electron kinetics respectively. Neglecting $\hat{b}$ in Eq. (1) yields the commonly adopted ideal TAE equation. ${ }^{1,2,4,6,7}$ For $\epsilon=0, \mathrm{Eq}$. (1) reduces to the equation for the kinetic Alfvén wave in slab geometry. ${ }^{12-14}$ Although the coefficients of the last two terms of Eq. (1) are small, these two terms should not be treated as mere perturbations because they may (and, indeed do) create new branch of eigenmodes.

In the $2 \mathrm{D}$ system of $\mathrm{Eq}$. (1), the ballooning symmetry, i.e., the invariance under the translation $x \rightarrow x+1, \ell \rightarrow \ell+1$, is violated by the radial dependence of $f(x)$. To solve this problem with broken ballooning symmetry, we shall adopt a systematic procedure based on the 2D ballooning transform. For the toroidal Alfvén waves, it is appropriate to consider $\Omega^{2} f(x) \equiv 1+\epsilon g(x)$ with $g(x) \approx g+2 x / m \hat{\epsilon}+\ldots$ where $\hat{\epsilon} \equiv \epsilon /\left[\partial \ln \left(q^{2} / v_{A}^{2}\right) / \partial \ln q^{2}\right]_{q=q_{0}}$, and $g$ is the $2 \mathrm{D}$-eigenvalue of order unity, measuring the frequency shift from the center of gap. Making use of the 2D ballooning transform ${ }^{19}$

$$
\phi_{\ell}(x)=\oint d \lambda d k \exp [i k(x-\ell)-i \lambda \ell] \widehat{\varphi}(k, \lambda)
$$

and neglecting higher order effects, we obtain a $2 D$ equation in the $k-\lambda$ representation

$$
\begin{aligned}
\frac{\partial^{2}}{\partial k^{2}} \varphi(k, \lambda) & +\frac{1+\epsilon \hat{g}}{4} \varphi(k, \lambda)+\frac{\epsilon}{2} \cos (k+\lambda) \varphi(k, \lambda)-\frac{s^{2}}{\left(1+s^{2} k^{2}\right)^{2}} \varphi(k, \lambda) \\
& -b\left(1+s^{2} k^{2}\right) \varphi(k, \lambda)=0
\end{aligned}
$$

for the scaled variable $\varphi \equiv \hat{\varphi} \sqrt{1+s^{2} k^{2}}$, which must satisfy the evanescent boundary condi- 
tion at large $k$, and the periodic boundary condition in $\lambda$. In Eq. (3), $b \equiv\left(\rho_{s} k_{\theta}\right)^{2} / 4+i \ldots$, and

$$
\widehat{g} \equiv g-(2 i / m \widehat{\epsilon}) \frac{\partial}{\partial \lambda}
$$

contains derivatives with respect to $\lambda$. Defining the self-adjoint ballooning operator

$$
\mathcal{L}[\lambda] \equiv \frac{\partial^{2}}{\partial k^{2}}+\frac{1+\epsilon \tilde{g}(\lambda)}{4}+\frac{\epsilon}{2} \cos (k+\lambda)-\frac{s^{2}}{\left(1+s^{2} k^{2}\right)^{2}}+b\left(1+s^{2} k^{2}\right)
$$

where $\tilde{g}(\lambda)$ is the parameterized ( $\lambda$--dependent) eigenvalue of $\mathcal{L}[\lambda]$, we rewrite Eq. (3) (correct to the first order of $\epsilon$ ) as

$$
\left[\left[\mathcal{L}[\lambda]+\frac{\epsilon}{4}(g-\tilde{g}(\lambda))-\frac{i \epsilon}{2 m \hat{\epsilon}} \frac{\partial}{\partial \lambda}\right] \varphi(k, \lambda)=0,\right.
$$

which will be solved perturbatively. Notice that in Eq. (5), we have neglected the higher order derivatives (with respect to $\lambda$ ) such as $\left(1 / m^{2}\right) \partial^{2} / \partial \lambda^{2}$ arising from the "higher order" symmetry breakings associated with $(x / m)^{2}$ or $(\ell / m)^{2} \ldots$ terms. This is possible only if $(1 / m) \partial / \partial \lambda$ is shown a posteriori to be proportional to a small parameter intrinsic to the system (here, the inverse of the aspect ratio $\epsilon$ ), so that the perturbative solution to the first order can be justified.

Following the methodology of Ref. 19, we choose $\chi(k, \lambda)$ to satisfy the zeroth order ballooning equation

$$
\mathcal{L}[\lambda] \chi(k, \lambda)=0
$$

for which the $\lambda$-dependence of $\chi$ comes merely through $\cos \lambda$ and $\sin \lambda$ [Eq. (4)]. The lowest order wave function is constructed to be $\Psi(\lambda) \chi(k, \lambda)$, where $\Psi(\lambda)$ is assumed to have a fast variation in $\lambda$. Assuming that the mode number $m \sim \epsilon^{-2}$, and expanding the total wave function

$$
\varphi(k, \lambda)=\Psi(\lambda) \chi(k, \lambda)+\varphi^{(1)}(k, \lambda)+\ldots
$$

as a perturbation series, we can readily obtain the first order equation

$$
\mathcal{L}[\lambda] \varphi^{(1)}+\frac{\epsilon}{4}[g-\tilde{g}(\lambda)] \Psi(\lambda) \chi(k, \lambda)-\frac{i \epsilon}{2 m \hat{\epsilon}} \frac{d \Psi(\lambda)}{d \lambda} \chi(k, \lambda)=0 .
$$


Due to the self-adjointness of the ballooning operator $\mathcal{L}$, standard manipulations reduce Eq. (8) to a first-order differential equation in $\lambda$

$$
\frac{2 i}{m \hat{\epsilon}} \frac{d \Psi(\lambda)}{d \lambda}-[g-\tilde{g}(\lambda)] \Psi(\lambda)=0,
$$

with the general solution

$$
\Psi(\lambda)=\exp \left(-\frac{i m \hat{\epsilon}}{2} \int^{\lambda} d \lambda^{\prime}\left[g-\tilde{g}\left(\lambda^{\prime}\right)\right]\right)
$$

The imposition of periodic boundary conditions on $\Psi(\lambda)[\lambda,(0,2 \pi)]$ determines both the 2Deigenvalue $g$, and the wave function $\Psi$ :

$$
g=\oint d \lambda \widetilde{g}(\lambda)-\frac{2 N}{m \hat{\epsilon}}
$$

where $N$ is an integer. It is clearly evident from Eqs. (10) and (11) that $\Psi(\lambda)$ is fast varying, but non-localized in $\lambda$-space, in contrast to the localized $\Psi(\lambda)$ of the conventional ballooning theory. ${ }^{15-19}$

We must also emphasize that, for the present case, the perturbative "expansion parameter" $(1 / m) \partial / \partial \lambda$ of the ballooning formalism comes out to be $\epsilon$, in lieu of $1 / \sqrt{n}$ for the conventional ballooning theory. For Alfvén waves in typical tokamaks, this parameter indeed happens to be smaller than unity justifying the relevance of our approach to the realistic scenario. This fortunate situation, in general, does not pertain; for weakly dissipative drift waves, for example, the effective $\epsilon$ (the coefficient of the toroidal term with appropriate normalization) turns out to be $2 q / s$, a quantity greater than unity throughout the machine. Thus the neglect of all higher order derivatives in Eq. (5) cannot be justified, and as a consequence, the non-localized $\Psi(\lambda)$ is unlikely $t$ be a proper description for drift waves unless some other realistic small parameter can be identified in the system. 


\section{2D-Eigenvalues and Mode Structure}

In the ideal limit the zeroth order ballooning equation $[\mathrm{Eq} .(6)]$ reduces to

$$
\left[\frac{\partial^{2}}{\partial k^{2}}+\frac{1+\epsilon \tilde{g}(\lambda)}{4}+\frac{\epsilon}{2} \cos (k+\lambda)-F(k)\right] x(k, \lambda)=0
$$

with $F(k) \equiv s^{2} /\left(1+s^{2} k^{2}\right)^{2}$. It has been shown that in the small $\epsilon$ limits, Eq. (12) can be manipulated to yield the analytic dispersion relation, ${ }^{7,10}$

$$
D_{1} \tilde{g}(\lambda)+D_{2} \sqrt{1-\tilde{g}^{2}(\lambda)}=\cos \lambda
$$

where $D_{1}$ and $D_{2}$ have been tabulated in Ref. 7 for various values of magnetic shear. In particular, for weak shear, both $D_{1}$ and $D_{2}$ are exponentially large, so that $\tilde{g}(\lambda)$ tends to be exponentially $\lambda$-independent. Or the other hand, as the magnetic shear becomes large, $D_{2}$ goes to zero very rapidly. As a result, the $2 \mathrm{D}$-eigenvalue, $g$, also goes to zero on averaging $\cos \lambda$ over $\lambda$.

In the general case, Eq. (13) can be solved for the physical $\tilde{g}(\lambda)$, and the result substituted in Eq. (11) to obtain the ideal eigenvalue

$$
g=-\frac{2 D_{2}}{\pi \sqrt{D_{1}^{2}+D_{2}^{2}}} E\left(\frac{1}{D_{1}^{2}+D_{2}^{2}}\right)
$$

where $E\left(\kappa^{2}\right) \equiv \int_{0}^{\pi / 2} d \phi \sqrt{1-\kappa^{2} \sin ^{2} \phi}$ is the complete elliptic integral of the second kind.

With the electron kinetics, the 2D-eigenvalues are obtained by numerical methods. We first solve [using a shooting code] the ballooning Eq. (6) to obtain the parameterized eigenvalue $\tilde{g}(\lambda)$. The numerically obtained $\tilde{g}(\lambda)$ is then averaged over $\lambda$ to yield the kinetic eigenvalue $g$, which now contains damping. For the TAE branch, the $\lambda$ dependence of the real (imaginary) part of $\widetilde{g}(\lambda)$ is displayed in Fig. 1a [Fig. 1b] for various values of the shear parameter $s=0.2,0.5$, and 1.0. with $\epsilon=0.2$. The complex parameter $b$ is represented by its absolute value $|b|$ and the phase $\operatorname{Arg} b \equiv \tan ^{-1}(\operatorname{Im} b / \operatorname{Re} b)$, for which we use $|b|=6 \times 10^{-5}$ 
and $\operatorname{Arg} b=-0.2$. The $\lambda$-dependence of $\tilde{g}$ is qualitatively similar to that described by the analytic dispersion, Eq. (13) for the ideal mode, i.e., it is very weak at small shear, and tends to be oscillatory around zero for large shear. The large damping rate for $\lambda=\pi$ in the present ballooning calculation would make the average damping rate to be quite different from the lowest $(\lambda=0)$ damping rate predicted by the conventional ballooning approach. This intrinsic 2D effect will have important consequences for the stability of TAE. For the KTAE branch the $\lambda$-dependence of $\tilde{g}$ is much weaker than that for TAE, and $\operatorname{Re} g$ is never near zero. These two features suggest the one-dimensional origin of the mode, and indicate that the effects of multi-gap coupling on KTAE are expected to be weak.

The result of Figs. $1 \mathrm{a}$ and $\mathrm{lb}$ also indicates that $\operatorname{Re} g$ is much greater than $\operatorname{Im} g$. As a result, $\Psi(\lambda)$ is essentially a purely oscillatory function of $\lambda$. This implies a non-ballooned mode structure in poloidal direction, even if the magnetic shear is not small. The radial extension of this non-ballooned mode is measured by $\Delta r \sim \epsilon r$ with a suppression factor arising from the weak $\lambda$-dependence of $\tilde{g}$ either for the small magnetic shear or for the KTAE branch.

The 2D-eigenvalues for TAE (KTAE) are plotted in Figs. 2a, 2b (Figs. 3a, 3b) as functions of the parameter $|b| s^{2}$, which measures the strength of the kinetic term. For all these cases $\epsilon=0.2$, and the shear values of $0.2,0.5$, and 1.0 label the curves $a, b$ and $c$ respectively. In addition, the curves with the subscript 2 are obtained from the present theory which naturally includes the multi-gap coupling, whereas the curves with subscript 1 are from the single-gap theory for comparison. In Sec. IV, we will show that the single-gap theory of Ref. 11 is a limiting case of the present theory. Large shear pushes the Re $g$ of TAE towards zero, i.e., towards the center of the gap. However, the shear effect on the real eigenvalues of KTAE, which resides in the continuum, is not as it is strong as for the TAE.

The magnetic shear augments damping rates for both TAE and KTAE, and stronger for KTAE. However, the TAE suffers stronger damping than KTAE in regions of parameters 
where kinetic effects are moderately strong; i.e., for high temperature, moderate shear and moderate mode numbers. It may be thus easier to excite KTAE than TAE in the interesting parameter regimes. This tendency is demonstrated in Fig. 4, where we find that as $|b|$ increases, there is a critical value (dependent on $s$ ) beyond which $\mathrm{KTAE}$ is less damped than TAE.

The strong kinetic damping on TAE is also related to the kinetic effects on the mode structure. The ideal TAE mode extension in the $k$-space, primarily measured by $k \sim 4 / \epsilon \sqrt{1-g^{2}}$, could be greatly modified by the electron kinetics that tends to capture the mode in a much less extended $k$ region. This mechanism for the enhancement of damping rate due to electron kinetics requires an intrinsic inclusion of the kinetics; a simple-minded perturbative estimate presuming ideal mode structure will not do. These conclusions are supportive, and are in essential qualitative agreement with the basic results of the single gap theory of Ref. 11.

\section{Transit to Single-Gap Theory}

In the small shear limit, the ballooning equation [Eq. (6)] can be converted to a solvable system by taking averages over the fast scale arising from toroidicity. Defining $\varphi \equiv$ $\varphi_{c} \cos (k / 2)+\varphi_{s} \sin (k / 2)$, and averaging Eq. (6) [over $k$ ] weighted with $\sin k / 2$ and $\cos k / 2$, one obtains a set of coupled equations for $\varphi_{c}$, and $\varphi_{s} .{ }^{1}$ These two equations can be expressed in terms of a new pair of fields $\varphi_{ \pm} \equiv \varphi_{c} \pm i \varphi_{s}$ :

$$
\begin{aligned}
& \left(\frac{d^{2}}{d \bar{k}^{2}}+i \frac{d}{d \bar{k}}+h(\bar{k})\right) \varphi_{-}=-\frac{\epsilon}{4} \exp (i \lambda) \varphi_{+}, \\
& \left(\frac{d^{2}}{d \bar{k}^{2}}-i \frac{d}{d \bar{k}}+h(\bar{k})\right) \varphi_{+}=-\frac{\epsilon}{4} \exp (-i \lambda) \varphi_{-},
\end{aligned}
$$

where $\bar{k}$ denotes the slow scale distinct from the fast scale $k$, and $h(\bar{k}) \equiv \epsilon g / 4-F(\bar{k})-b(1+$ $\left.s^{2} \bar{k}^{2}\right)$. The explicit $\lambda$ dependence has no effect on the eigenvalue determined by Eqs. (15),(16), because $\exp ( \pm i \lambda)$ can be absorbed into the wave amplitudes $\varphi_{ \pm}$. For example, one can intro- 
duce $\bar{\varphi}_{+} \equiv \varphi_{+} \exp (i \lambda)$, so that both the $\bar{\varphi}_{+}$and $\varphi_{-}$are $\lambda$-independent. This $\lambda$-independence of the averaged equation is not surprising, for $\lambda$ is just a phase shift, which is effectively averaged along with the average over the fast scale. For averaged equations, the scale length of $\bar{k}$ is much greater than unity due to its slow variation, so that the second derivatives can be neglected. Then, the coupled equations (15) and (16) are reduced to two first order differential equations, the basic equations derived under the single-gap assumption, ${ }^{11}$ except that in $h$ the $G$-term of Ref. 11 is replaced by the $F$-term. As $s \bar{k} \gg 1$ (for small but finite $b, s \bar{k} \sim(s / b)^{1 / 3} \gg 1$ ), there exist only two independent parameters (the coefficients of the $F$-term and the kinetic term) that may enter into the dispersion. For the KTAE branch, since the $F$-term is not crucial, only one independent parameter $\left(4 s \rho_{s} \bar{k}_{\theta} / \epsilon\right)^{2}\left(\equiv \hat{\tau} / \hat{\epsilon}^{3}\right.$ of Ref. 11) may come into the dispersion. This is just the KTAE scaling of Ref. 11.

It is worth noting that the small shear limit implies a weak $\lambda$-dependence for the parameterized eigenvalue $\tilde{g}(\lambda)$ of the ballooning equation. Since the exponential factor of Eq. (10) is then suppressed by the weak variation of $\widetilde{g}(\lambda)$, the wave function $\Psi(\lambda)$ can no longer vary on a fast scale. This relative $\lambda$-dependence of $\Psi$ implies that now the entire wave function in the $k-\lambda$ space is described by $\chi(k, \lambda)$ alone. The averaging scheme then yields a mode structure $\chi \sim \bar{\varphi}_{+}(\bar{k}) \exp (-i \lambda-i k / 2)+\varphi_{-}(\bar{k}) \exp (i k / 2)$. Substituting this expression into the $2 \mathrm{D}$-transform $\left[\mathrm{Eq}\right.$. (2)], one can readily find that $\bar{\varphi}_{+}$stands for $\ell=-1$ component, and $\varphi$ - stands for $\ell=0$ component; both these components are peaked at $x=-1 / 2$. The delineation of the reduction to the single-gap theory is thus complete. Physically, the weak shear implies that the separation between the gaps becomes large and the wave function localized in these gaps do not overlap.

Quantitative comparison of the theory with multi-gap coupling, to the single-gap theory is also presentcd in Figs. 2a, 2b (Figs. 3a, 3b) for TAE (KTAE). The numerical results for the single-gap theory are obtained by solving Eqs. (15) and (16) with the second derivative terns $\left(d^{2} / d \bar{k}^{2}\right)$ neglected. For magnetic shear near 1.0 the theory with multi-gap coupling 
predicts a stronger damping rate than that given by the single-gap theory (by a factor of $\simeq$ 2) for the TAE branch. However, the effect from multi-gap coupling is not significant for the KTAE branch. The discrepancy between the two theories is noticeable for large shear and tends to become negligible as the magnetic shear becomes small.

\section{Acknowledgments}

This work was supported by the U.S. Department of Energy contract \#DE-FG05-80ET53088. 


\section{References}

1. C.Z. Cheng, L. Chen, and M.S. Chance, Annals of Physics (N.Y.) 161, 21 (1985).

2. C.Z. Cheng and M.S. Chance, Phys. Fluids 29, 3695 (1986).

3. S. Riyopoulos and S.M. Mahajan, Phys. Fluids 29, 731 (1986).

4. G.Y. Fu and J.W. Van Dam, Phys. Fluids B 1, 1949 (1989).

5. K.L. Wong et al., Phys. Rev. Lett. 66, 1874 (1991); W.W. Heidbrink, E.J. Strait, E. Doyle, G. Sager, and R. Snider, Nucl. Fusion 31, 1635 (1991).

6. F. Zonca and L. Chen, Phys. Rev. Lett. 68, 592 (1992).

7. M.N. Rosenbluth, H.L. Berk, J.W. Van Dam, and D.M. Lindberg, Phys. Rev. Lett. 68, 596 (1992).

8. H.L. Berk, J.W. Van Dam, Z. Guo, and D.M. Lindberg, Phys. Fluids B 4, 1806 (1992).

9. N.N. Gorelenkov and S.E. Sharapov, IAEA Technical Committee Meeting on Alpha Particles in Fusion Research, Aspenas Sweden (June 10-14, 1991).

10. R. Dewar and Y.Z. Zhang, Bull. Am. Phys. Soc. (1992).

11. R.R. Mett and S.M. Mahajan, Phys. Fluids B 4, 2885 (1992).

12. D.W. Ross, G.L. Chen, and S.M. Mahajan, Phys. Fluids 25, 652 (1982).

13. S.M. Mahajan, Phys. Fluids 27, 2238 (1984).

14. A. Hasegawa and L. Chen, Phys. Fluids 19, 1924 (1976).

15. J.W. Connor, R.J. Hastie, and J.B. Taylor, Proc. R. Soc. A 1, 365 (1979). 
16. Y.C. Lee and J.W. Van Dam, in Proc. Finite beta theory workshop, arenna Summer School of Plasma Physics, eds. B. Coppi and B. Sadowski (U.S. Department of Energy, Office of Fusion Energy, CONF-7709167 (1979).

17. A. Glasser, ibid. p. 55 .

18. R. Dewar, Theory of Fusion Plasma, Proceedings of the workshop held at Villa CipressiVarenna, Italy, Aug. 24-28, 1987. ed. by A. Bondeson, E. Sindoni, and F. Troyon (Editrice Compositori Societa Italiana di Fisica, Bologna, Italy, 1988), p. 107.

19. Y.Z. Zhang and S.M. Mahajan, Phys. Lett. A 157, 133 (1991).

20. Y.Z. Zhang, S.M. Mahajan, and X.D. Zhang, Phys. Fluids B 4, 2729 (1992). 


\section{Figure Captions}

la(1b). The real (imaginary) parameterized eigenvalue of the TAE mode as the numerical solution of Eq. (6). The curves $a, b$, and $c$, stand for $s=0.2,0.5$, and 1.0 respectively with $|b|=6 \times 10^{-5}, \operatorname{Arg} b=-0.2$ and $\epsilon=0.2$.

$2 \mathrm{a}(2 \mathrm{~b})$. The real (imaginary) 2D-eigenvalue of the TAE mode vs. $|b| s^{2}$ with $\epsilon=0.2$. The curves $a, b, c$ and $d$ stand for $s=0.2,0.5,1.0$ and 2.0 respectively. The subscripts 1 and 2 represent the results from the single-gap and multi-gap theory respectively.

$\left.3 \mathrm{a}_{1}^{\prime} 3 \mathrm{~b}\right)$. The real (imaginary) 2D-eigenvalue of the KTAE mode for the same scenario of Figs. $2 \mathrm{a}$ and $2 \mathrm{~b}$.

4. The comparison of the damping rate $\left(-\operatorname{Im} g\right.$ ) for TAE (Curves $T_{a}-T_{c}$ ) and KTAE (Curves $K_{a}-K_{c}$ ) for the same scenario of Fig. 2b. The subscripts $a, b$, and $c$ stand for $s=0.2,0.5$, and 1.0 respectively. 

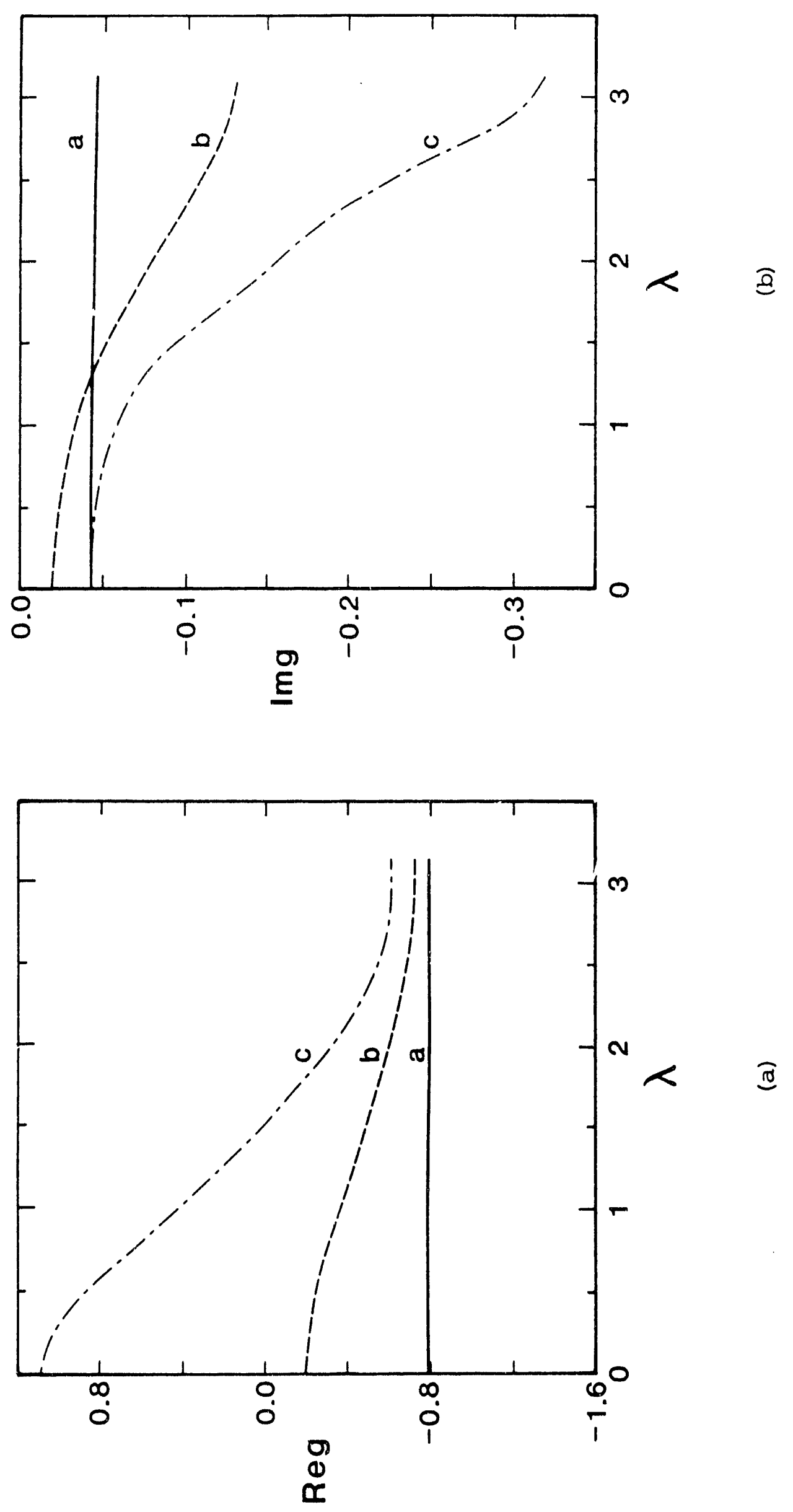

Fig. 1 


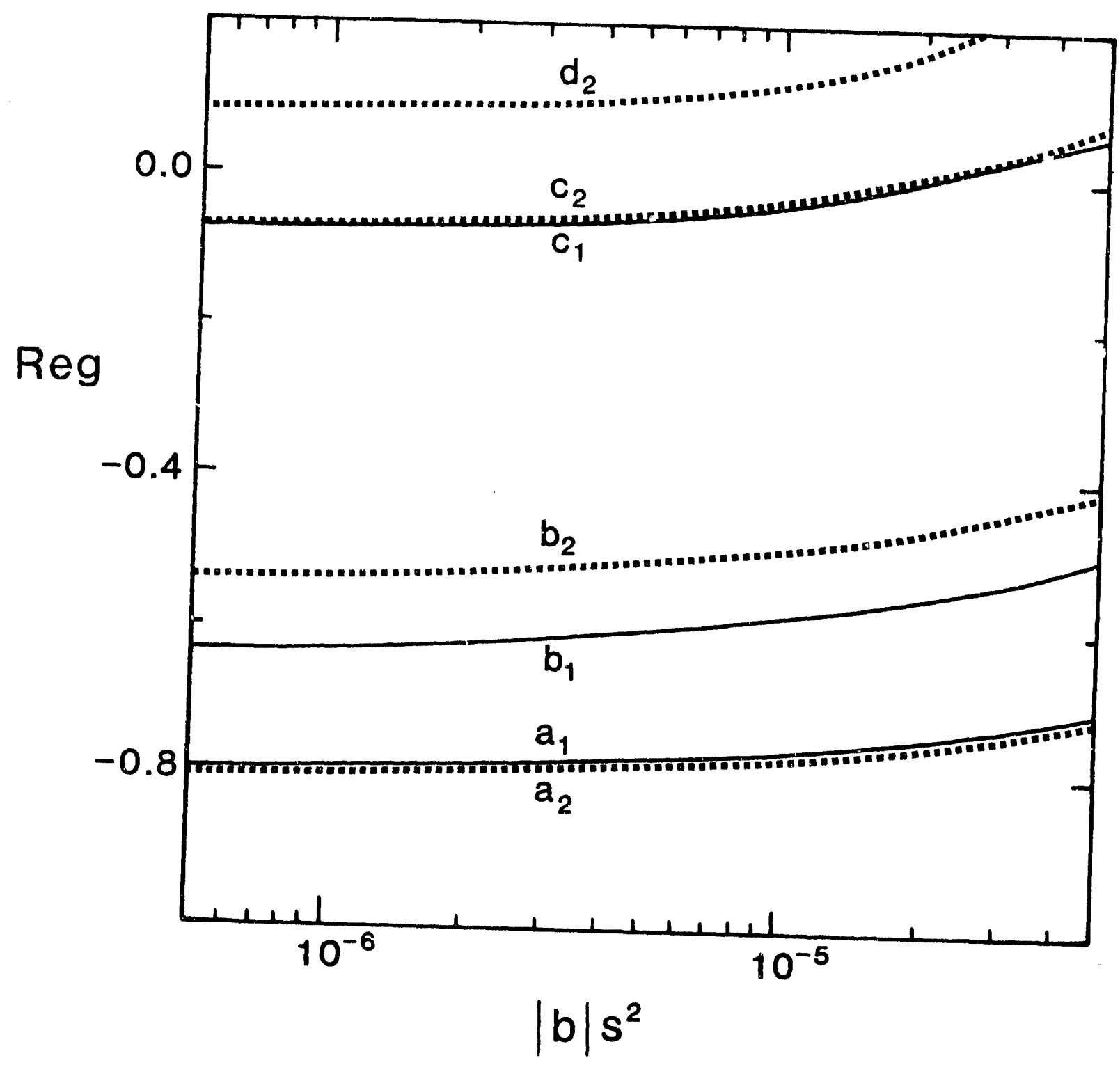

Fig. $2 a$ 


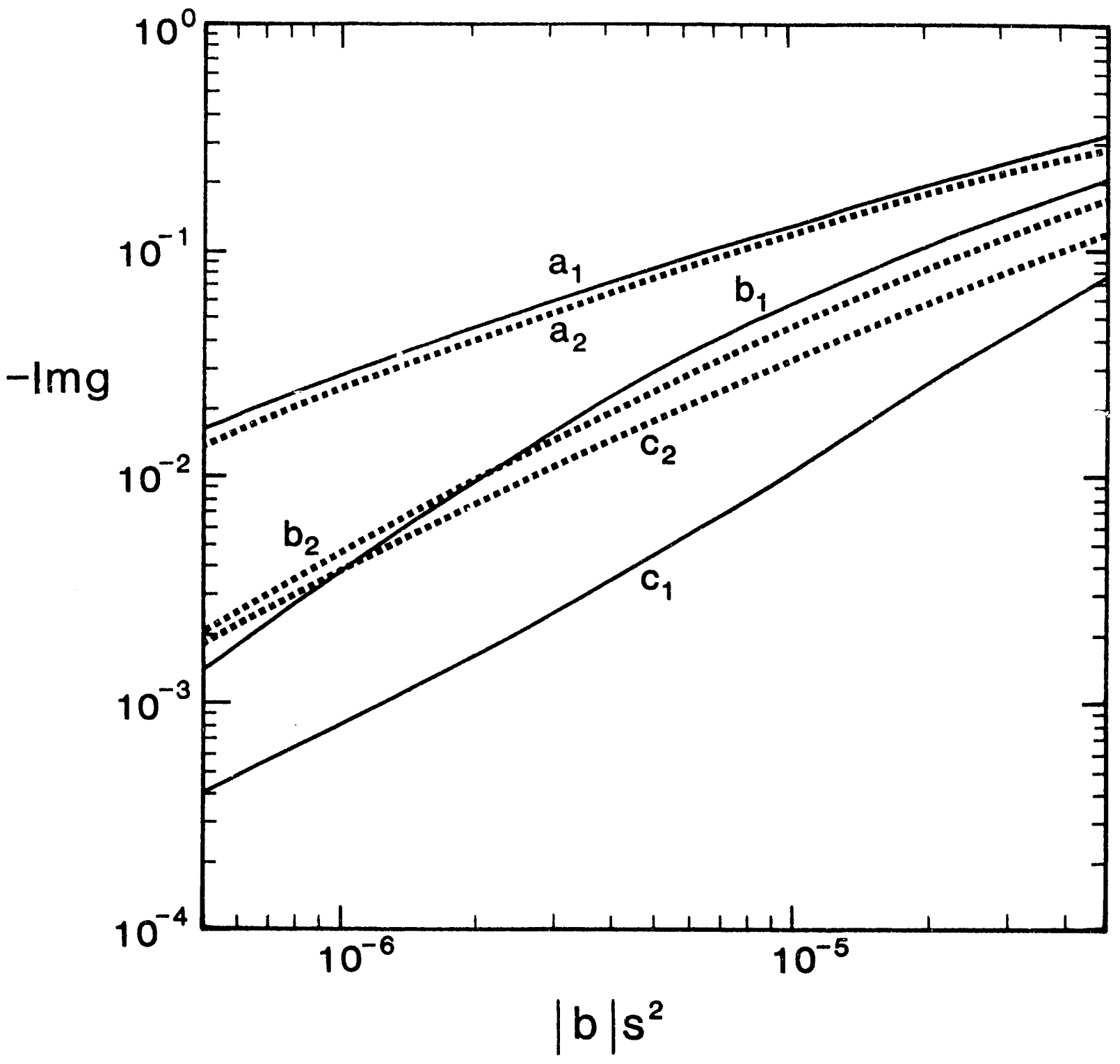

Fig. 2b 


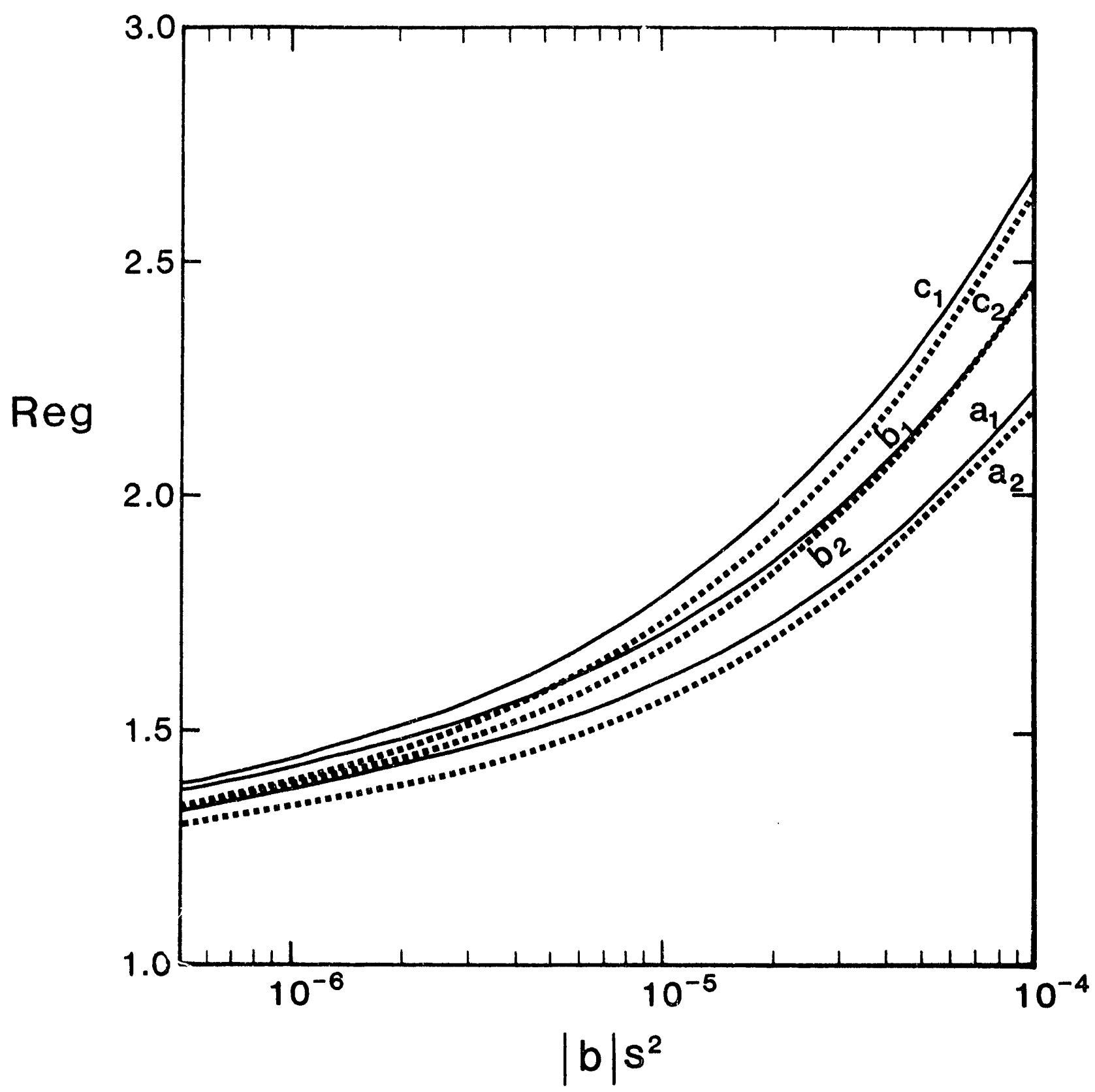

Fig. $3 a$ 


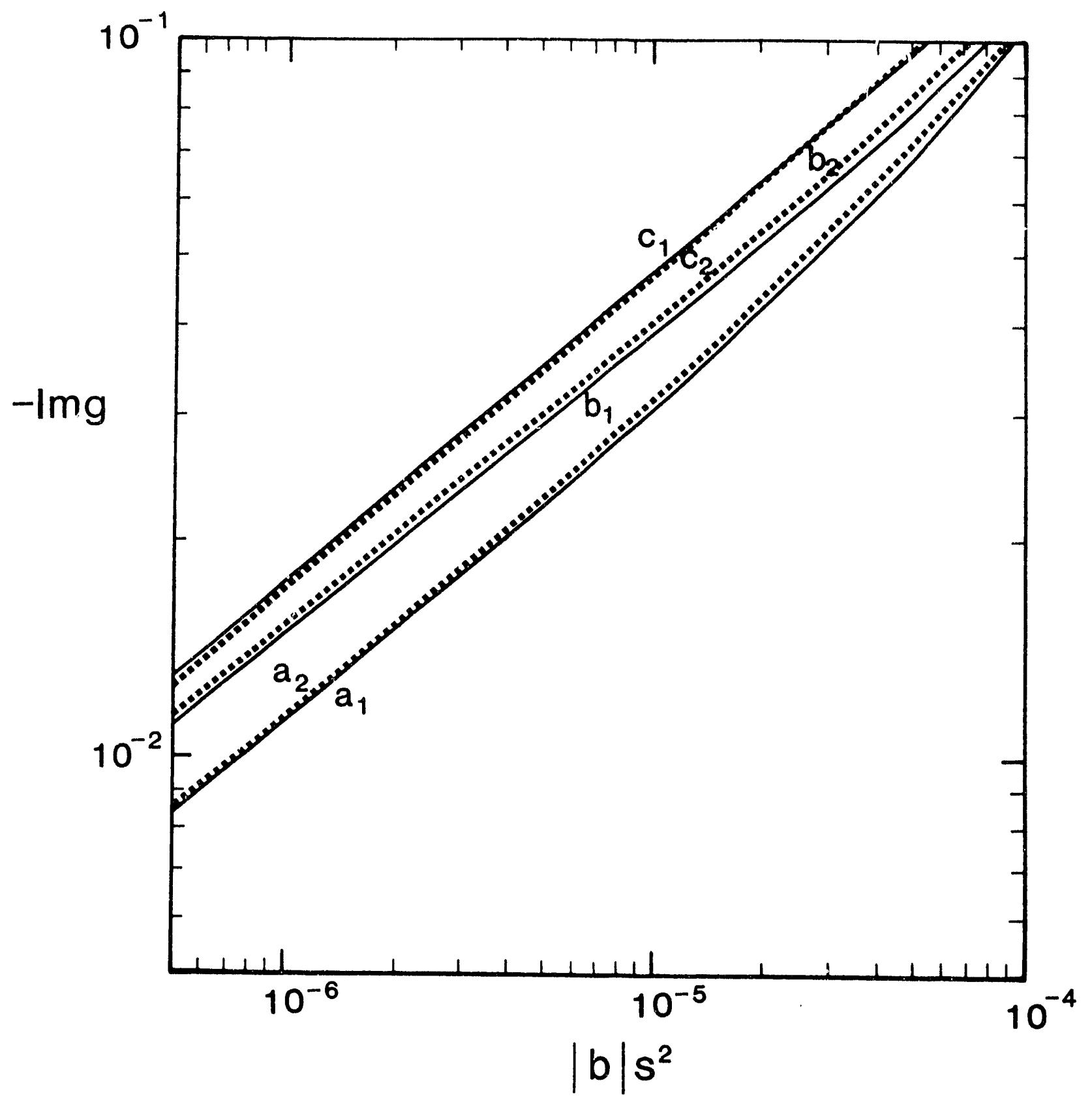

Fig. $3 b$ 


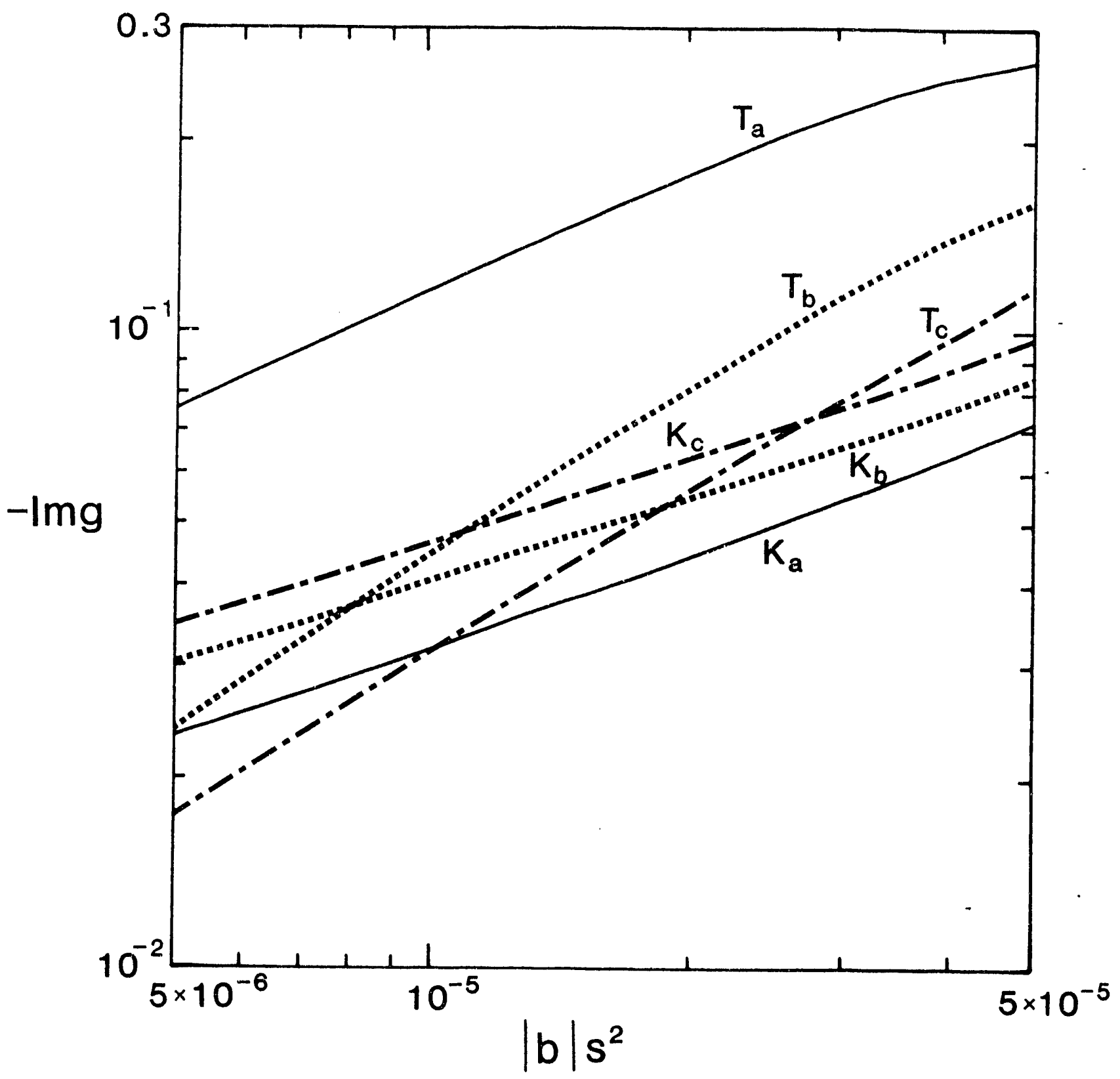

Fig. 4 

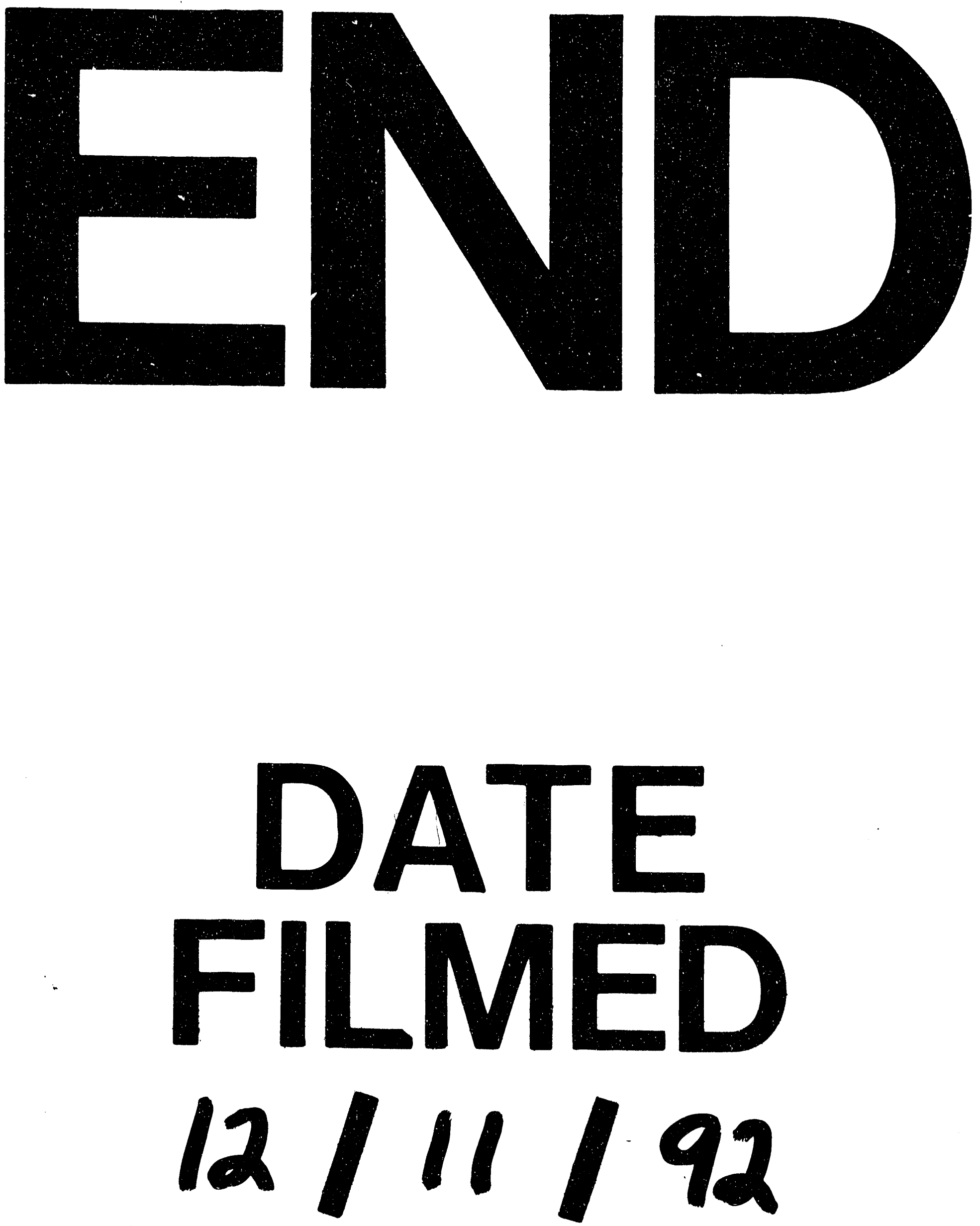
